\title{
Engineering the electronic, magnetic, and gap-related properties of the quinternary half-metallic Heusler alloys
}

K. Özdoğan, E. Şaşığlu, and I. Galanakis

Citation: Journal of Applied Physics 103, 023503 (2008);

View online: https://doi.org/10.1063/1.2831224

View Table of Contents: http://aip.scitation.org/toc/jap/103/2

Published by the American Institute of Physics

\section{Articles you may be interested in}

Conditions for spin-gapless semiconducting behavior in $\mathrm{Mn}_{2} \mathrm{CoAl}$ inverse Heusler compound Journal of Applied Physics 115, 093908 (2014); 10.1063/1.4867917

Antiferromagnetic half-metals, gapless half-metals, and spin gapless semiconductors: The $\mathrm{D}_{3}$-type Heusler alloys

Applied Physics Letters 103, 232409 (2013); 10.1063/1.4840318

Slater-Pauling behavior in LiMgPdSn-type multifunctional quaternary Heusler materials: Half-metallicity, spingapless and magnetic semiconductors

Journal of Applied Physics 113, 193903 (2013); 10.1063/1.4805063

$\mathrm{Ab}$ initio electronic and magnetic properties of half-metallic NiCrSi and NiMnSi Heusler alloys: The role of defects and interfaces

Journal of Applied Physics 104, 083916 (2008); 10.1063/1.3005882

High TC half-metallic fully-compensated ferrimagnetic Heusler compounds

Applied Physics Letters 99, 052509 (2011); 10.1063/1.3619844

Search for spin gapless semiconductors: The case of inverse Heusler compounds

Applied Physics Letters 102, 022402 (2013); 10.1063/1.4775599

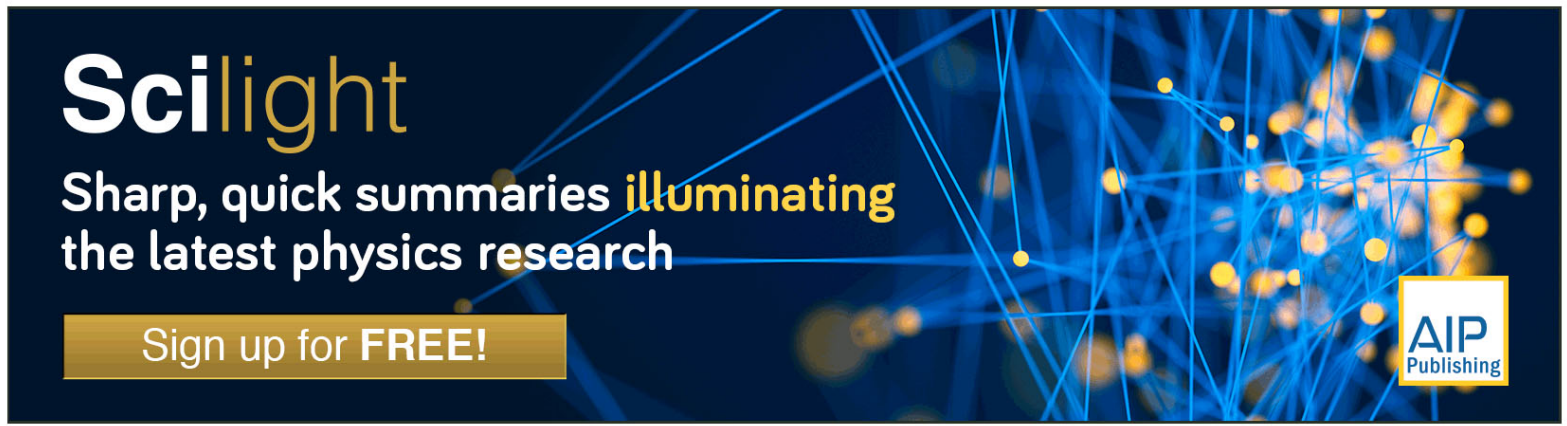




\title{
Engineering the electronic, magnetic, and gap-related properties of the quinternary half-metallic Heusler alloys
}

\author{
K. Özdoğan, ${ }^{1, a)}$ E. Şaşıŏlu, ${ }^{2, b)}$ and I. Galanakis ${ }^{3, c)}$ \\ ${ }^{1}$ Department of Physics, Gebze Institute of Technology, Gebze, 41400, Kocaeli, Turkey \\ ${ }^{2}$ Institut für Festkörperforschung, Forschungszentrum Jülich, D-52425 Jülich, Germany \\ and Department of Physics, Fatih University, 34500, Büyükçekmece, Ístanbul, Turkey \\ ${ }^{3}$ Department of Materials Science, School of Natural Sciences, University of Patras, \\ GR-26504 Patra, Greece
}

(Received 6 September 2007; accepted 12 November 2007; published online 17 January 2008)

\begin{abstract}
We study the electronic and magnetic properties of the quinternary full Heusler alloys of the type $\mathrm{Co}_{2}\left[\mathrm{Cr}_{1-x} \mathrm{Mn}_{x}\right]\left[\mathrm{Al}_{1-y} \mathrm{Si}_{y}\right]$ employing three different approaches: (i) the coherent potential approximation; (ii) the virtual crystal approximation; and (iii) supercell calculations. All three methods give similar results, and the local environment manifests itself only for small details of the density of states. All alloys under study are shown to be half-metals, and their total spin moments follow the so-called Slater-Pauling behavior of the ideal half-metallic systems. We especially concentrate on the properties related to the minority-spin band gap. We present the possibility to engineer the properties of these alloys by changing the relative concentrations of the low-valent transition metal and $s p$ atoms in a continuous way. Our results show that for realistic applications, compounds rich in $\mathrm{Si}$ and $\mathrm{Cr}$ are ideal since they combine large energy gaps (around $0.6 \mathrm{eV}$ ), robust half-metallicity with respect to defects (the Fermi level is located near the middle of the gap), and high values of the majority-spin density of states around the Fermi level, which are needed for large values of the perfectly spin-polarized current in spintronic devices like spin valves or magnetic tunnel junctions. (c) 2008 American Institute of Physics. [DOI: 10.1063/1.2831224]
\end{abstract}

\section{INTRODUCTION}

Rapid growth of the magnetoelectronics field, also known as spintronics, brought to the attention of scientists new phenomena. ${ }^{1-3}$ One of the most interesting concepts in spintronics is half-metallicity. ${ }^{4-10}$ Half-metals are hybrids between normal metals and semiconductors. The majority-spin band is crossed by the Fermi level as in a normal metal, while the Fermi level falls within a gap in the minority-spin band as in semiconductors, leading to a perfect $100 \%$ spinpolarization at the Fermi level. ${ }^{5}$ Such compounds should be able to produce a fully spin-polarized current and be ideal spin injectors into a semiconductor, thus maximizing the efficiency of spintronic devices. ${ }^{11}$ de Groot and his collaborators in 1983 predicted the existence of half-metallicity in the case of the intermetallic semi-Heusler alloy $\mathrm{NiMnSb}^{12}$ and the origin of the gap seems to be well understood. ${ }^{13,14}$ The half-metallic character of $\mathrm{NiMnSb}$ in single crystals seems to have been well-established experimentally. Infrared absorption ${ }^{15}$ and spin-polarized positron annihilation ${ }^{16}$ gave a spin polarization of $\sim 100 \%$ at the Fermi level. Firstprinciples electronic structure calculations explained the origin of half-metallicity in Heusler alloys in terms of the hybridization between the $d$-orbitals of the transition metal atoms and, as it was shown, the half-metallicity is also closely related to the total spin magnetic moment in the unit cell. ${ }^{13}$ However, in many other experiments on low-

\footnotetext{
${ }^{a)}$ Electronic mail: kozdogan@gyte.edu.tr.

b) Electronic mail: e.sasioglu@fz-juelich.de.

c)Electronic mail: galanakis@upatras.gr.
}

dimensional structures, half-metallicity has not been found since the properties measured were surface- and interfacesensitive.

Although semi-Heuslers initially monopolized most interest in this topic, lately interest has shifted to the so-called full-Heusler compounds like $\mathrm{Co}_{2} \mathrm{MnAl}$. Webster synthesized such alloys in $1971,{ }^{17}$ and almost 20 years later it was argued in two papers by a Japanese group that they should be half-metals. ${ }^{18}$ The emergence of the field of magnetoelectronics again brought these alloys to the center of scientific interest, ${ }^{19,20}$ and first-principles calculations have explained the origin of half-metallicity, demonstrating also that the perfect half-metallic full Heusler alloys show so-called SlaterPauling behavior. The total spin moments in the unit cell, $M_{t}$ in $\mu_{B}$, equals the total number of valence electrons in the unit cell minus 24 , since there are exactly 12 occupied minorityspin states. ${ }^{20}$ This rule is of interest because it provides an easy way to connect half-metallicity with the total spinmoment in the unit cell, which is easily determined using experiments such as superconducting quantum interference device measurements. Although several full-Heusler alloys have been shown to be half-metallic in their bulk form, ${ }^{20,21}$ $a b$ initio calculations have shown that the surfaces ${ }^{22}$ and interfaces of the full-Heusler compounds ${ }^{23}$ loose their halfmetallicity (Hashemifar and co-workers have shown that it is possible to restore half-metallicity at some surfaces). ${ }^{24}$ Interface state, temperature-driven excitations ${ }^{25-27}$ and defects ${ }^{28,29}$ seem to destroy half-metallicity (vacancies keep in some cases the half-metallic character of the parent compound). ${ }^{30}$ Other important aspects of these alloys, such as their orbital magnetism, ${ }^{31}$ doping, ${ }^{32}$ structural stability, ${ }^{33}$ the appearance 
of ferrimagnetism ${ }^{34-36}$ and antiferromagnetism, ${ }^{37}$ and the interplay of exchange interactions, ${ }^{38,39}$ have been addressed in literature.

Over the last two years interest in full-Heusler alloys containing cobalt has been focused on the so-called quaternary Heusler alloys, which are found to present halfmetallicity as long as the corresponding perfect parent compounds are half-metals. ${ }^{40,41}$ Several authors studied the properties of $\mathrm{Co}_{2}\left[\mathrm{Cr}_{1-x} \mathrm{Fe}_{x}\right] \mathrm{Al}$ as a function of the concentration $x .^{42,43}$ Moreover, the $\mathrm{Co}_{2}\left[\mathrm{Mn}_{1-x} \mathrm{Fe}_{x}\right] \mathrm{Si}$ alloys ${ }^{44}$ were extensively studied because $a b$ initio calculations, including the on-site Coulombic repulsion (the so-called Hubbard $U$ ), have shown that $\mathrm{Co}_{2} \mathrm{FeSi}$ can reach a total spin magnetic moment of $6 \mu_{B}$, which is the largest known spin moment for a half-metal. ${ }^{45,46}$ In a recent paper ${ }^{41}$ the authors employed a full-potential nonorthogonal local-orbital minimum-basis band structure scheme (FPLO) $)^{47,48}$ based on the local-spindensity approximation ${ }^{49}$ to study the properties of the quaternary Heusler compounds, and the $\mathrm{Co}_{2}\left[\mathrm{Cr}_{1-x} \mathrm{Mn}_{x}\right] Z$ ( $Z$ $=\mathrm{Al}, \mathrm{Ga}, \mathrm{Si}, \mathrm{Ge}$, or $\mathrm{Sn}$ ) compounds were predicted to be half-metallic for all concentrations.

In this manuscript we investigate the possibility to allow for an additional degree of freedom and study the quinternary Heusler alloys where also the chemical elements at the $Z$ site are mixed. We have decided to focus on the $\mathrm{Co}_{2}\left[\mathrm{Cr}_{1-x} \mathrm{Mn}_{x}\right]\left[\mathrm{Al}_{1-y} \mathrm{Si}_{y}\right]$ compounds because all four parent compounds are half-metals and such compounds seem feasible experimentally. Our main interest is to find a way to engineer in a continuous way the properties of these alloys related with the gap, i.e., the width of the minority-spin band gap, the position of the Fermi level, and the majority-spin density of states at the Fermi level.

\section{DESCRIPTION OF PRESENT CALCULATIONS}

In our calculations we have used the experimental lattice constants for the perfect compounds containing $\mathrm{Mn},{ }^{50,51}$ $0.5756 \mathrm{~nm}$ for $\mathrm{Co}_{2} \mathrm{MnAl}$ and $0.565 \mathrm{~nm}$ for $\mathrm{Co}_{2} \mathrm{MnSi}$, and considered that the lattice constant varies linearly with the concentration $y$ of the $\mathrm{Al}$ and $\mathrm{Si}$ atoms. We assumed that the substitution of $\mathrm{Cr}$ for $\mathrm{Mn}$ does not change the lattice constant, since there is no data regarding the exact behavior of the lattice. This tactic is different than the one used in Ref. 40 , where it was assumed that the lattice constant varies linearly with the concentration $x$ of the transition-metal atom, but both methods give lattice constants within less than $1 \%$ difference and thus, practically identical results.

To perform our calculations we employed three different formalisms. First, the coherent potential approximation (CPA), initially developed by Blackman and collaborators in $1971{ }^{52}$ In 1997 Koepernik et al. extended this formalism and implemented it in a linear combination of atomic orbitals (LCAO) method, ${ }^{47}$ and later they expanded it and incorporated it in the FPLO method. ${ }^{48}$ This was made possible due to the localized character of the orbitals in this method, which allows for a single-site representation of the space. In CPA each $Y$ (or $Z$ ) site is occupied by both $\mathrm{Cr}$ and $\mathrm{Mn}$ (or $\mathrm{Al}$ and $\mathrm{Si}$ ) atoms, with a probability given by the respective concentration of each chemical type. Second, we used the
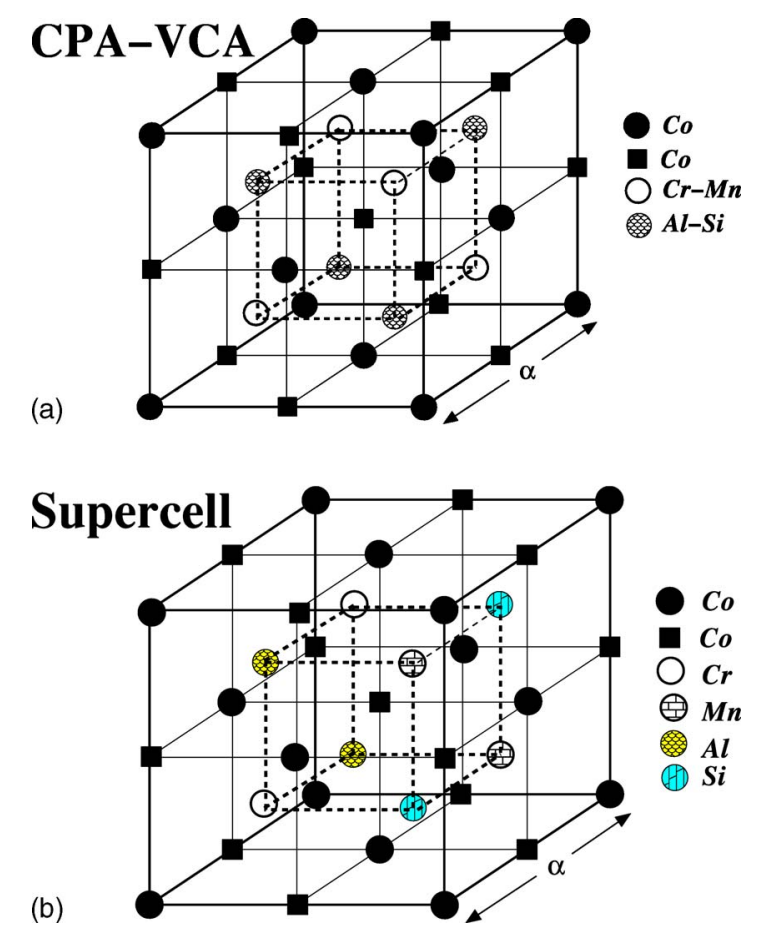

FIG. 1. (Color online) Schematic representation of the structure used for the $\mathrm{Co}_{2}\left[\mathrm{Cr}_{0.5} \mathrm{Mn}_{0.5}\right]\left[\mathrm{Al}_{0.5} \mathrm{Si}_{0.5}\right]$ alloy. In the upper panel is the structure used for the CPA and VCA calculations. In CPA the $\mathrm{Cr}-\mathrm{Mn}$ site is occupied by $\mathrm{Cr}$ atoms with a probability of $50 \%$ and by $\mathrm{Mn}$ atoms with a probability of $50 \%$. The Al-Si site is also occupied by both $\mathrm{Al}$ and $\mathrm{Si}$ atoms with $50 \%$ probability for each one. In VCA, the Cr-Mn site is occupied by a pseudoatom with a fractional number of valence electrons (24.5 electrons) and the $\mathrm{Al}-\mathrm{Si}$ site by a pseudoatom with 13.5 valence electrons. The unit cell for both CPA and VCA calculations contains four sites. In the lower panel we present the structure for the supercell calculations, where we take a double unit cell with respect to VCA and CPA containing eight atoms $\left(\mathrm{Co}_{4} \mathrm{CrMnAl}\right.$ compound). In the case where $x$ and/or $y$ take 0.25 or 0.75 as a value we have a unit cell with 16 atoms for the supercell calculations. Note also that there are two inequivalent Co sites in all cases which have the same environment rotated by $\pi / 2$.

so-called virtual crystal approximation (VCA). In VCA for the $Y$ (or $Z$ ) site we substitute both $\mathrm{Co}$ and $\mathrm{Mn}$ atoms (or $\mathrm{Al}$ and $\mathrm{Si}$ atoms) with an atom with a fractional number of electrons $(1-x) z^{\mathrm{Cr}}+x z^{\mathrm{Mn}}\left[\right.$ or $\left.(1-y) z^{\mathrm{Al}}+y z^{\mathrm{Si}}\right]$, where $z^{\mathrm{Cr}}$ is the number of electrons of $\mathrm{Cr}$ and similarly for the other chemical elements. Obviously in VCA, only the total spin magnetic moment in the unit cell and the total density of states (DOS) have a physical meaning, since we cannot project the properties of the pseudoatom on the different chemical types. We represent the structures within both CPA and VCA in the upper part of Fig. 1. The lattice is fcc, with four atoms as a basis set along the diagonal: two $\mathrm{Co}$ atoms, one $\mathrm{Cr}-\mathrm{Mn}$ site and one Al-Si site. Both CPA and VCA do not take into account the short-range interactions, since the real crystal is replaced by one where each $Y$ or $Z$ site contains two different chemical-type atoms. In reality, this is not true and we performed supercell (SC) calculations to examine this effect. We present the structure in the lower part of Fig. 1 for $x=0.5$ and $y=0.5$, where the unit cell contains eight atoms; in reality the correct chemical formula is $\mathrm{Co}_{2} \mathrm{CrMnAlSi}$. In the case where either of $x$ or $y$ is 0.25 or 0.75 , the unit cell contains 16 atoms. To compare between the three different approaches, 


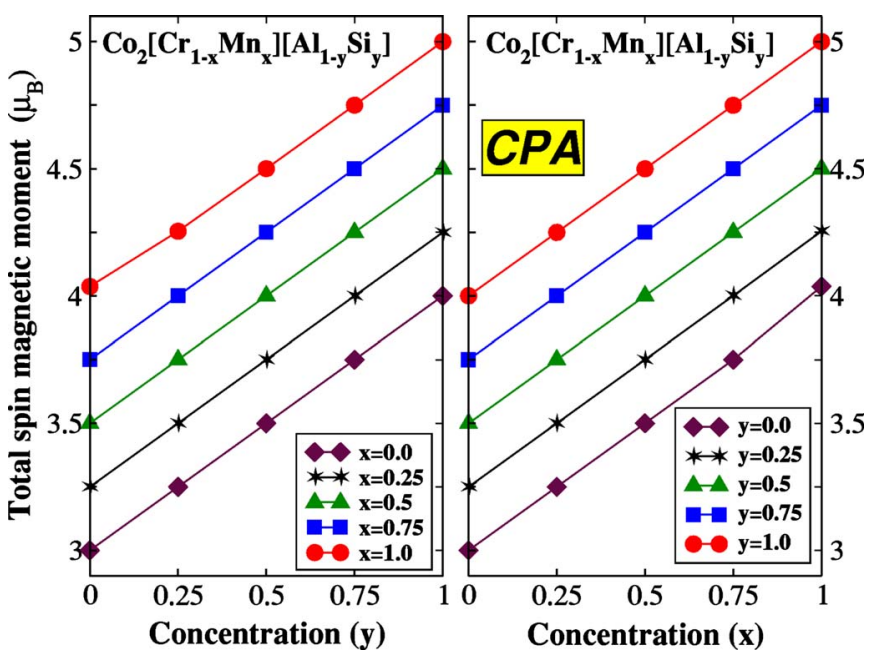

FIG. 2. (Color online) (Left) Total moment in $\mu_{B}$ as a function of the concentration $y$ in $\mathrm{Co}_{2}\left[\mathrm{Cr}_{1-x} \mathrm{Mn}_{x}\right]\left[\mathrm{Al}_{1-y} \mathrm{Si}_{y}\right]$ using the CPA method. Different lines correspond to constant values of $x$. (Right) Similar to the left panel as a function of $x$ with different lines corresponding to constant values of $y$. All compounds are half-metals showing Slater-Pauling behavior.

when we present the total DOS or the total spin magnetic moments, we always scale to a formula unit of four atoms.

Such an investigation of the gap-related properties of the Heusler alloys is of interest due to the large variety of applications of these alloys. Several experiments have been devoted to the study of the structural and magnetic properties of the quaternary Heusler alloys, ${ }^{53}$ and such films have been incorporated both in magnetic tunnel junctions ${ }^{54}$ and spin valves. ${ }^{55}$ Quinternary half-metallic full-Heusler alloys will provide an additional tool to tune the properties of these films and enhance the performance of the devices based on such films.

\section{TOTAL AND ATOM-RESOLVED SPIN MAGNETIC MOMENTS}

We begin our discussion on the quinternary alloys by discussing the magnetic spin moments. To present our results we discuss the trends as a function of the concentration $x$ of the transition-metal atoms $\mathrm{Cr}$ and $\mathrm{Mn}$, keeping the concentration $y$ of the $s p$ atoms constant, and afterward as a function of $y$ keeping $x$ constant. This presentation allows for an easier discussion of the results. In Fig. 2 we have plotted the total spin moment in the unit cell in $\mu_{B}$ within the CPA approach. If the alloys under study are half-metals, they should follow the Salter-Pauling behavior for the total spin moments, $M_{t}: M_{t}=Z_{t}-24 .{ }^{20}$ The total spin moment in $\mu_{B}$ is just the number of uncompensated spins and thus, the number 24 arises from the 12 occupied minority spin states (for details see Ref. 20). $Z_{t}$ denotes the total mean number of valence electrons and is given by the expression $Z_{t}=2 z^{\mathrm{Co}}$ $+(1-x) z^{\mathrm{Cr}}+x z^{\mathrm{Mn}}+(1-y) z^{\mathrm{Al}}+y z^{\mathrm{Si}}$, where $z$ denotes the valence of each atom. As presented in Fig. 2, the values of the total spin moments are spotted on top of straight lines, both when we plot the total moment as a function of $y$ (left panel) or as a function of $x$ (right panel). A close look at the figure reveals that all these straight lines follow the Slater-Pauling behavior for the full-Heusler alloys. $\mathrm{Co}_{2} \mathrm{CrAl}$ has 27 elec-
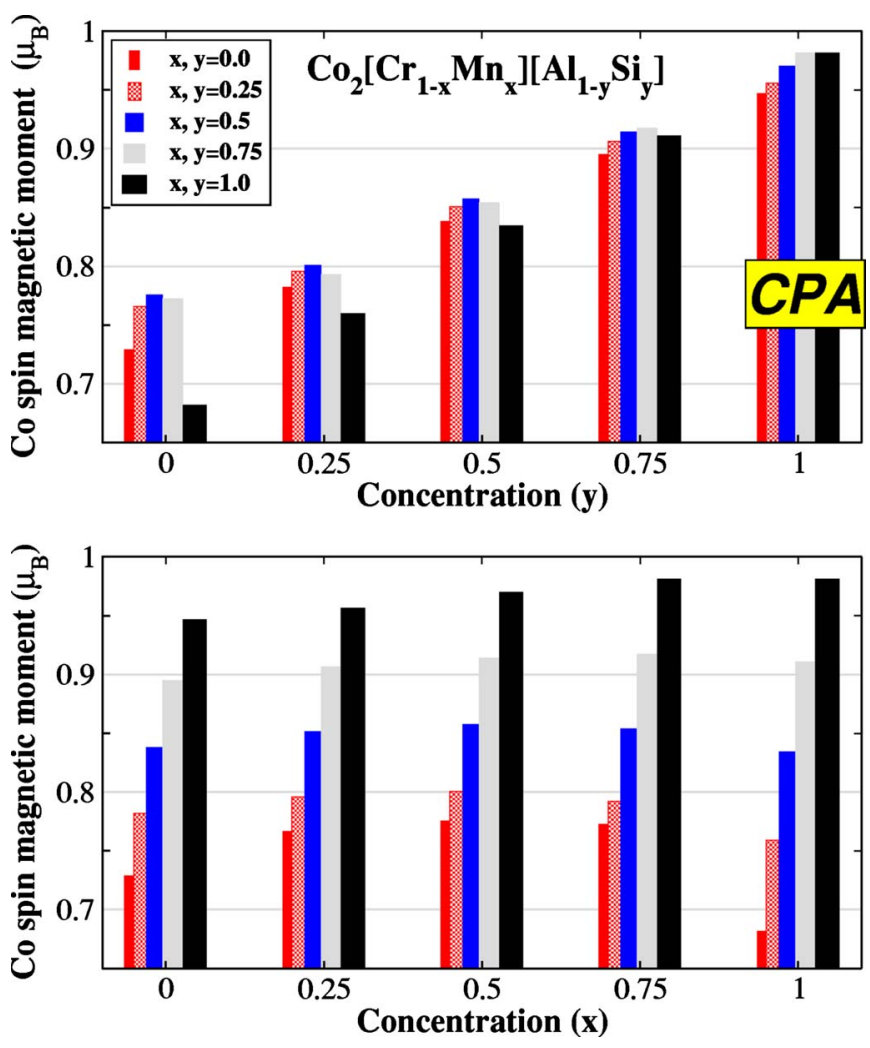

FIG. 3. (Color online) (Top) Co-resolved spin magnetic moment in $\mu_{B}$ as a function of $y$ concentration in $\mathrm{Co}_{2}\left[\mathrm{Cr}_{1-x} \mathrm{Mn}_{x}\right]\left[\mathrm{Al}_{1-y} \mathrm{Si}_{y}\right]$ using the CPA method. Different bars correspond to different values of $x$. (Bottom) Similar to the top panel as a function of $x$ with different bars corresponding to different values of $y$.

trons and an ideal total spin moment of $3 \mu_{B}$, which coincides with the calculated one. When we substitute $25 \%$ of the $\mathrm{Cr}$ atoms by $\operatorname{Mn}(x=0.25)$, we increase the total spin moment by $0.25 \mu_{B}$ each time, reaching a value of $4 \mu_{B}$ for $\mathrm{Co}_{2} \mathrm{MnAl}$. Also at each step when we substitute $25 \%$ of the $\mathrm{Si}$ atoms for $\mathrm{Al}$, again we increase the number of valence electrons by 0.25 and thus, the total spin moment increases also by $0.25 \mu_{B}$ at each step, reaching $4 \mu_{B}$ for $\mathrm{Co}_{2} \mathrm{CrSi}$ and $5 \mu_{B}$ for $\mathrm{Co}_{2} \mathrm{MnSi}$.

In Figs. 3-5 we have plotted the atom-resolved spin moments for the transition metal atoms $\mathrm{Co}, \mathrm{Cr}$, and $\mathrm{Mn}$, respectively, within the CPA approximation. In each figure we present the spin magnetic moments as a function of the concentration $y$ in the upper panel, with different bars corresponding to different $x$ values and as a function of $x$ in the lower panel with different bars corresponding to different values of $y$. We do not present the spin moments of the $s p$ atoms, since they are very small and negative (around $\left.-0.1 \mu_{B}\right)$. The negative sign arises from the $p$ states. The minority bonding $p$ states are completely occupied, while the majority bonding ones extend much more in energy crossing the Fermi level, resulting in a excess of minority $p$ states with respect to the majority ones and thus a negative spin moment. The behavior of the $s p$ atoms is extensively discussed in Refs. 5 and 20 and is independent of the $x$ and $y$ concentration.

For the Co spin moments in Fig. 3, it is obvious from the upper panel that the Co spin moment depends on the $y$ con- 

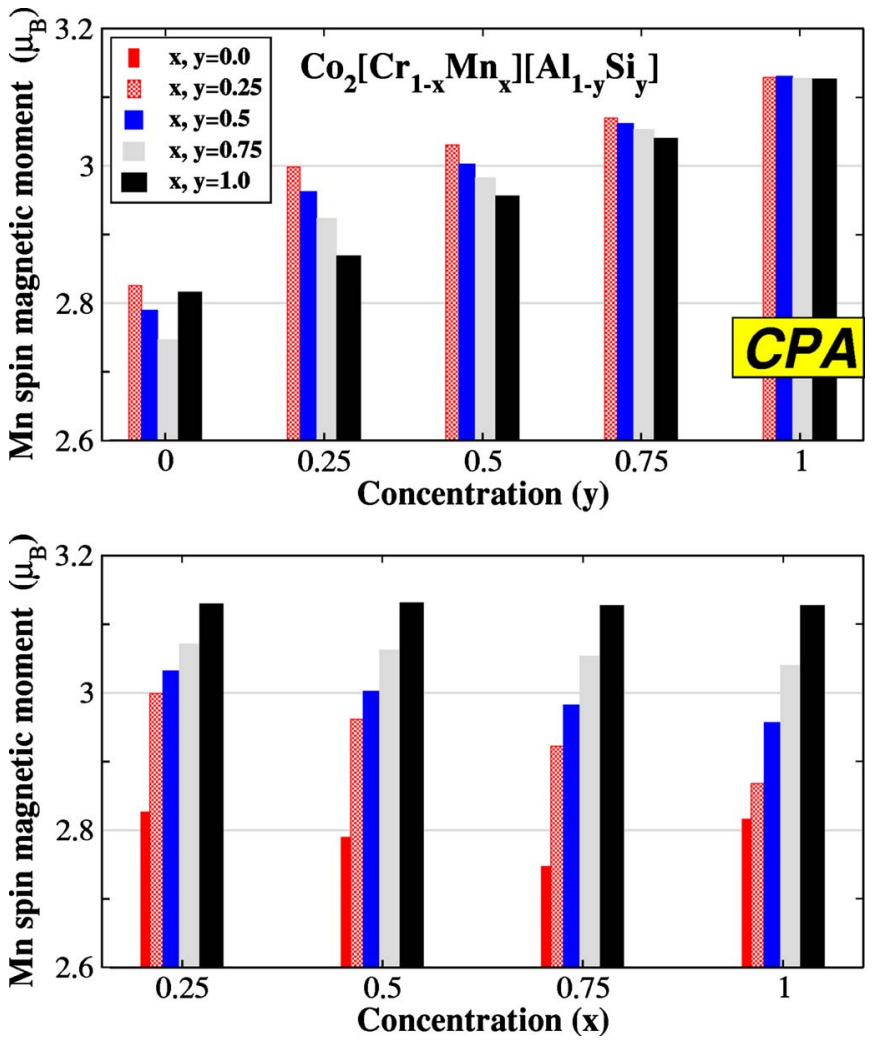

FIG. 4. (Color online) Same as Fig. 3 for the Mn atoms. The spin magnetic moment has been scaled to one Mn atom.

centrations of the $s p$ atoms. When $y=0$ and the compounds contain only $\mathrm{Al}$, the Co spin moments are quite small, around $0.7-0.75 \mu_{B}$. When $25 \%$ of the $\mathrm{Al}$ atoms are substituted by $\mathrm{Si}$, the Co spin moments increase and reach $0.8 \mu_{B}$. When $y=0.5$ the spin moments are around $0.85 \mu_{B}$, and when $y$ $=1$ and the compounds contain only $\mathrm{Si}$, the Co spin moments are around $0.95 \mu_{B}$. Thus, in general, as we increase the concentration of $\mathrm{Si}$ atoms by $25 \%$ the $\mathrm{Co}$ spin moment increases by $0.05 \mu_{B}$. Obviously the concentration of the $\mathrm{Cr}$ and $\mathrm{Mn}$ atoms does not affect the Co spin moment, as can be seen in Fig. 3. The substitution of $\mathrm{Cr}$ by $\mathrm{Mn}$ does not change the hybridization of the $d$-orbitals of the Co atoms with the $d$-orbitals of the lower valence transition metal atoms, $\mathrm{Cr}$ and Mn, and thus, the Co spin moment is not affected by the $x$ concentration. On the other hand, when we change the concentrations $1-y$ and $y$ of the $\mathrm{Al}$ and $\mathrm{Si}$ atoms, the extra electronic charge occupies majority spin electronic states of the transition metal atoms, as in a rigid band model, and increases the spin moment of all transition-metal atoms Co, $\mathrm{Cr}$, and $\mathrm{Mn}$.

In Figs. 4 and 5 we present the spin moments of the $\mathrm{Cr}$ and $\mathrm{Mn}$ atoms scaled to one atom. The conclusions drawn for the Co spin magnetic moments hold generally also for the $\mathrm{Mn}$ and $\mathrm{Cr}$ atoms, although the fluctuations are more important than for the $\mathrm{Co}$ atoms. Both $\mathrm{Cr}$ and $\mathrm{Mn}$ spin magnetic moments depend mainly on the concentration $1-y$ and $y$ of the $\mathrm{Al}$ and $\mathrm{Si}$ atoms. The $\mathrm{Mn}$ spin moment starts from around $2.8 \mu_{B}$ for $y=0$, corresponding to the compounds containing only $\mathrm{Al}$, and goes up to $3.1 \mu_{B}$ for $y=1$, corresponding to the compounds where only $\mathrm{Si}$ is present. Similarly, the $\mathrm{Cr}$ spin
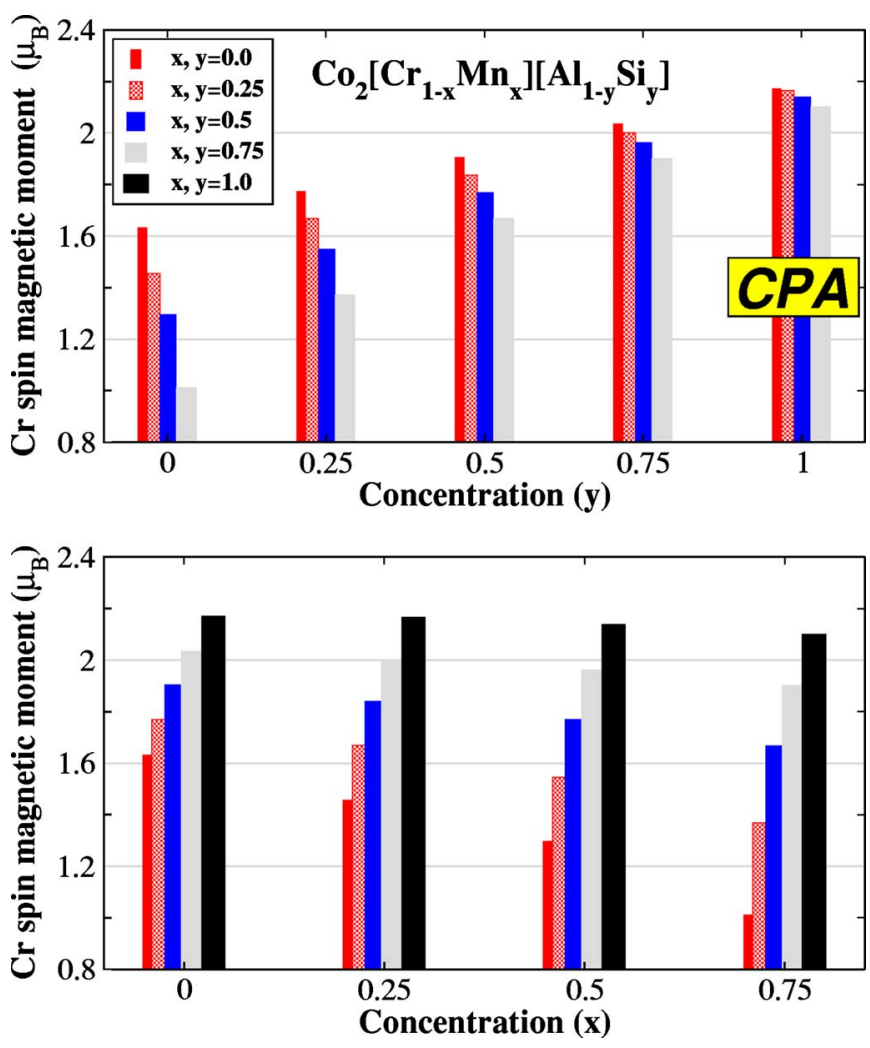

FIG. 5. (Color online) Same as Fig. 3 for the $\mathrm{Cr}$ atoms. The spin magnetic moment has been scaled to one $\mathrm{Cr}$ atom.

moments reach a value of around $2.1 \mu_{B}$ for the exclusively Si-based alloys $(y=1)$. When $y=0$ or $y=0.25$ the $\mathrm{Cr}$ spin moment presents large fluctuations with respect to the $x$ concentration being $1.6 \mu_{B}$ for $\mathrm{Co}_{2} \mathrm{CrAl}$ and $0.9 \mu_{B}$ for $\mathrm{Co}_{2}\left[\mathrm{Cr}_{0.75} \mathrm{Mn}_{0.25}\right] \mathrm{Al}$. This behavior of the $\mathrm{Cr}$ spin moments has been explained in Ref. 34, where it was attributed to the pinning of the Fermi level in a narrow band created by the $\mathrm{Cr}$ triple-degenerated majority $t_{2 g}$ electrons, which overlaps strongly with the double-degenerated $e_{g}$ majority states, contrary to the behavior of the Mn atoms. ${ }^{35}$ As shown in Ref. 34, a small shift of the Fermi level results in large changes of the $\mathrm{Cr}$ spin moments. When we increase the concentration in $\mathrm{Si}$ atoms, the extra electrons push the $\mathrm{Cr}$ majority states lower in energy. Thus, the $\mathrm{Cr}$ spin magnetic moment is insensitive to the exact position of the Fermi level, and the $\mathrm{Cr}$ spin moment is the same for all compounds with different $x$ value but the same $y$ value when $y=0.5,0.75$, or 1.0 (compounds rich in $\mathrm{Si}$ ).

Until now we have discussed the spin moments resulting for the CPA approach. In Table I we have gathered the total and spin magnetic moments from all three SC, CPA, and VCA calculations as a function of the concentrations $x$ and $y$. For the SC we scaled the moment to a unit cell of four atoms in order to compare them with the other two methods, although for the calculations we used a unit cell of 8 or 16 atoms, depending on the values of the $x$ and $y$ concentrations. Our first remark concerns the total spin magnetic moments in the third column. We have included just one value, since all three methods predict for all compounds the halfmetallic character and thus, a total spin moment in agree- 
TABLE I. Calculated spin magnetic moments in $\mu_{B}$ using a supercell construction for $\mathrm{Co}_{2}\left[\mathrm{Cr}_{1-x} \mathrm{Mn}_{x}\right]$ $\times\left[\mathrm{Al}_{1-y} \mathrm{Si}_{y}\right]$ as a function of the concentration of $x$ and $y$. We do not present the spin moments of the $\mathrm{Al}$ and $\mathrm{Si}$ atoms since they are very small (around $-0.10 \mu_{B}$ for all compounds). All compounds are half-metals and the total spin moment is the ideal one predicted by the Slater-Pauling rule. (We scaled the total spin moment to the elementary unit cell containing four atoms only; the unit cell in the supercell calculations contains either 8 or 16 atoms.) In the case where there are more than one inequivalent atoms of the same chemical kind, we present the largest spin moment; the difference between this moment and the other moments of the same chemical type are less than $0.05 \mu_{B}$ in all cases. In parenthesis are the calculated spin magnetic moments using the CPA approximation and in brackets using the VCA approximation. Note that within VCA we have a pseudoatom with 24.5 electrons instead of distinct Co and Mn atoms, and we include these spin moments in both the Co and Mn columns.

\begin{tabular}{cccccc}
\hline \hline & & & $m^{\mathrm{Co}}$ & $m^{\mathrm{Cr}}$ & $m^{\mathrm{Mn}}$ \\
$x$ & $y$ & $m^{\text {Total }}$ & $\mathrm{SC}(\mathrm{CPA})[\mathrm{VCA}]$ & $\mathrm{SC}(\mathrm{CPA})[\mathrm{VCA}]$ & $\mathrm{SC}(\mathrm{CPA})[\mathrm{VCA}]$ \\
\hline 0.00 & 0.00 & 3.00 & $0.73(0.73)[0.73]$ & $1.63(1.63)[1.63]$ & - \\
0.00 & 0.25 & 3.25 & $0.78(0.78)[0.79]$ & $1.77(1.76)[1.76]$ & - \\
0.00 & 0.50 & 3.50 & $0.84(0.84)[0.84]$ & $1.90(1.90)[1.90]$ & - \\
0.00 & 0.75 & 3.75 & $0.90(0.89)[0.90]$ & $2.02(2.03)[2.03]$ & - \\
0.00 & 1.00 & 4.00 & $0.95(0.95)[0.95]$ & $2.17(2.17)[2.17]$ & - \\
0.25 & 0.00 & 3.25 & $0.73(0.77)[0.68]$ & $1.54(1.45)[1.99]$ & $2.98(2.83)[1.99]$ \\
0.25 & 0.25 & 3.50 & $0.78(0.80)[0.76]$ & $1.71(1.67)[2.09]$ & $2.97(3.00)[2.09]$ \\
0.25 & 0.50 & 3.75 & $0.88(0.85)[0.82]$ & $1.78(1.84)[2.22]$ & $2.95(3.03)[2.22]$ \\
0.25 & 0.75 & 4.00 & $0.90(0.91)[0.87]$ & $1.99(2.00)[2.35]$ & $3.12(3.07)[2.35]$ \\
0.25 & 1.00 & 4.25 & $0.95(0.96)[0.92]$ & $2.19(2.16)[2.49]$ & $3.10(3.13)[2.49]$ \\
0.50 & 0.00 & 3.50 & $0.72(0.78)[0.66]$ & $1.42(1.29)[2.30]$ & $2.92(2.79)[2.30]$ \\
0.50 & 0.25 & 3.75 & $0.83(0.80)[0.72]$ & $1.53(1.55)[2.42]$ & $2.88(2.96)[2.42]$ \\
0.50 & 0.50 & 4.00 & $0.85(0.86)[0.79]$ & $1.77(1.77)[2.54]$ & $3.03(3.00)[2.54]$ \\
0.50 & 0.75 & 4.25 & $0.90(0.91)[0.85]$ & $1.99(1.96)[2.65]$ & $3.12(3.06)[2.65]$ \\
0.50 & 1.00 & 4.50 & $0.96(0.97)[0.91]$ & $2.18(2.14)[2.76]$ & $3.12(3.13)[2.76]$ \\
0.75 & 0.00 & 3.75 & $0.71(0.77)[0.63]$ & $1.18(1.00)[2.63]$ & $2.88(2.75)[2.63]$ \\
0.75 & 0.25 & 4.00 & $0.78(0.79)[0.71]$ & $1.47(1.37)[2.71]$ & $2.91(2.92)[2.71]$ \\
0.75 & 0.50 & 4.25 & $0.85(0.85)[0.80]$ & $1.76(1.67)[2.78]$ & $2.99(2.98)[2.78]$ \\
0.75 & 0.75 & 4.50 & $0.90(0.92)[0.87]$ & $2.04(1.90)[2.87]$ & $3.05(3.05)[2.87]$ \\
0.75 & 1.00 & 4.75 & $0.97(0.98)[0.93]$ & $2.20(2.10)[2.97]$ & $3.12(3.12)[2.97]$ \\
1.00 & 0.00 & 4.00 & $0.68(0.68)[0.68]$ & - & $2.82(2.82)[2.82]$ \\
1.00 & 0.25 & 4.25 & $0.75(0.76)[0.76]$ & - & $2.87(2.87)[2.87]$ \\
1.00 & 0.50 & 4.50 & $0.83(0.83)[0.83]$ & - & $2.98(2.96)[2.96]$ \\
1.00 & 0.75 & 4.75 & $0.91(0.91)[0.91]$ & - & $3.09(3.04)[3.04]$ \\
1.00 & 1.00 & 5.00 & $0.98(0.98)[0.98]$ & - & $3.13(3.13)[3.13]$ \\
\hline \hline & & & & & -
\end{tabular}

ment with the Slater-Pauling behavior. The most important feature of this table is the atom-resolved spin moments within the supercell calculations with respect to the CPA approach. SC takes into account the local effects (short-range interactions) contrary to CPA, which is a mean-field theory taking into account only the long-range order. The atomresolved spin magnetic moments are practically the same for all three transition metal elements, $\mathrm{Co}, \mathrm{Cr}$, and $\mathrm{Mn}$, regardless of the $x$ and $y$ concentrations within both CPA and SC. Thus, for the full-Heusler alloys under study the short-range interactions have a minimal influence on the electronic structure of these alloys, and these alloys can be accurately described by mean-field theories like CPA. This behavior should be attributed to their lattice structure, which is a close-packed one and presents very high symmetry in all directions. Their properties are largely governed by symmetry arguments (like the origin of the gap or the fixed number of minority occupied states), ${ }^{20}$ and substitution of an atom by a neighboring chemical element only marginally affects the properties of these compounds. This argument is also supported by the VCA calculations. The substitution of the crystal by a virtual one with atoms of fractional electronic charge gives the same total spin moment and almost identical Co spin moments with respect to both SC and the more sophisticated mean-field theory of CPA. Thus, Co atoms are not really sensitive to their local environment as long as the symmetry is not broken and each Co atom has four low-valence transition metal atoms and four $s p$ atoms as first neighbors. In Table I we include also the spin moments of the pseudoatoms within VCA, although they have no physical meaning.

\section{DENSITY OF STATES}

In the previous section we have discussed the halfmetallicity of the quinternary full-Heusler alloys in terms of the total spin magnetic moments. In this section we concentrate on the density of states. This is important since we will use the different DOS to compute in detail the width of the gap, the position of the Fermi level, and the majority-spin DOS at the Femi level. Thus, we should be able to accurately define the edges of the minority spin-gap. In VCA and SC calculations the gap is clearly defined but problems arise within CPA. When we compute the DOS within CPA we have to use a smearing for the DOS. In previous publications 


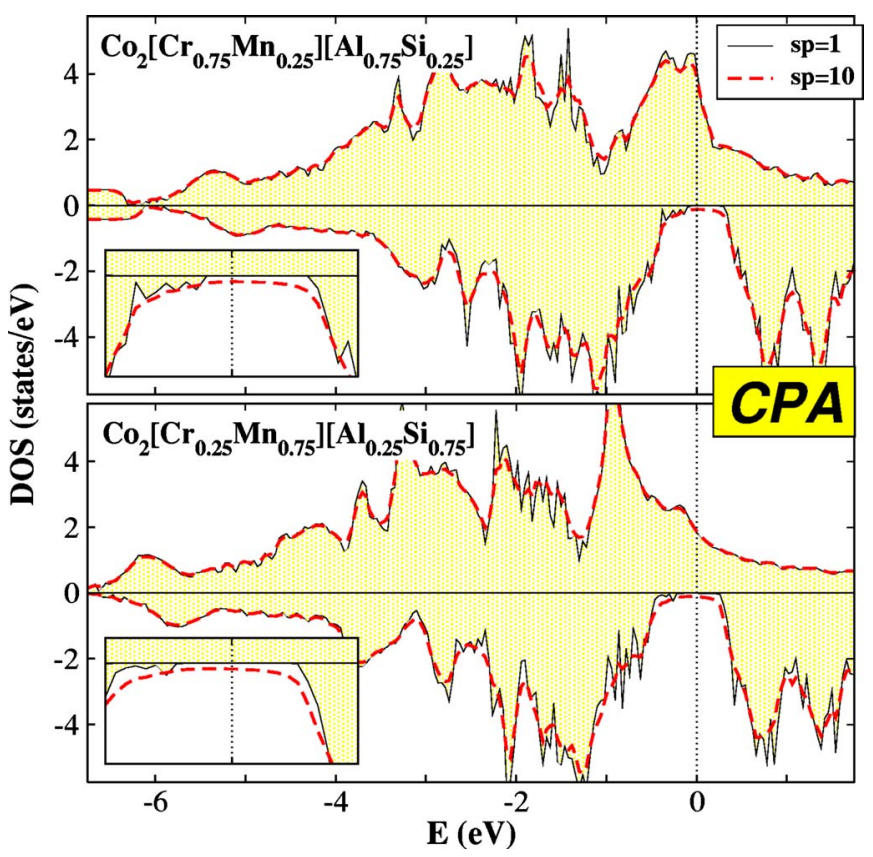

FIG. 6. (Color online) Total DOS in states/eV for two different compounds using the CPA method and a smearing of $10^{-4}$ hartree $(\mathrm{sp}=1)$ and a smearing of $10^{-3}$ hartree $(\mathrm{sp}=10)$. The Fermi level was chosen to be the zero of the energy axis.

with the same method (see Refs. 34 and 35, for example) we usually used a smearing of $10^{-3}$ hartree, but as shown in Fig. 6 for two different compounds, this yields a region of very low DOS instead of a real gap due to tails from both gap edges which overlap. Thus, such a large smearing is not suitable for the gap-related properties. If we use a smearing of $10^{-4}$ hartree, the calculation of DOS is much more tedious, but as shown in Fig. 6 we clearly get a region of zero DOS and we are able to define the edges of the gap. Thus, in all CPA calculations, which we will present in the following, we have used a broadening of $10^{-4}$ hartree.

In Fig. 7 we have plotted the total DOS for four different compounds, taking into account all possible combinations of $x$ or $y=0.25$ or 0.75 . All three methods, CPA, VCA, and SC, produce a similar total DOS (note that for SC we have scaled the DOS to a unit cell of four atoms). CPA and SC give the same positions for the bands of all atoms and the DOS almost coincide. VCA gives bands with the same mass center as CPA and SC but with different shape. This difference in shape with respect to CPA is expected, since VCA is an oversimplified mean-field approach. If we concentrate on the region around the gap, which we have blown up in the insets, it is obvious that all three methods give a similar description and similar values for the width of the gap and the position of the Fermi level. This will be analyzed in detail in the next section. These results support our statement in the previous section that short-range interactions are not important for the description of the magnetic and electronic properties of the half-metallic Heusler alloys when the disordered site contains atoms of neighboring chemical elements.

Finally, in Figs. 8 and 9 we plot the total and atomresolved DOS for the $\mathrm{Co}_{2}\left[\mathrm{Cr}_{0.5} \mathrm{Mn}_{0.5}\right]\left[\mathrm{Al}_{0.5} \mathrm{Si}_{0.5}\right]$ compounds within the CPA and SC approaches, respectively. All DOS
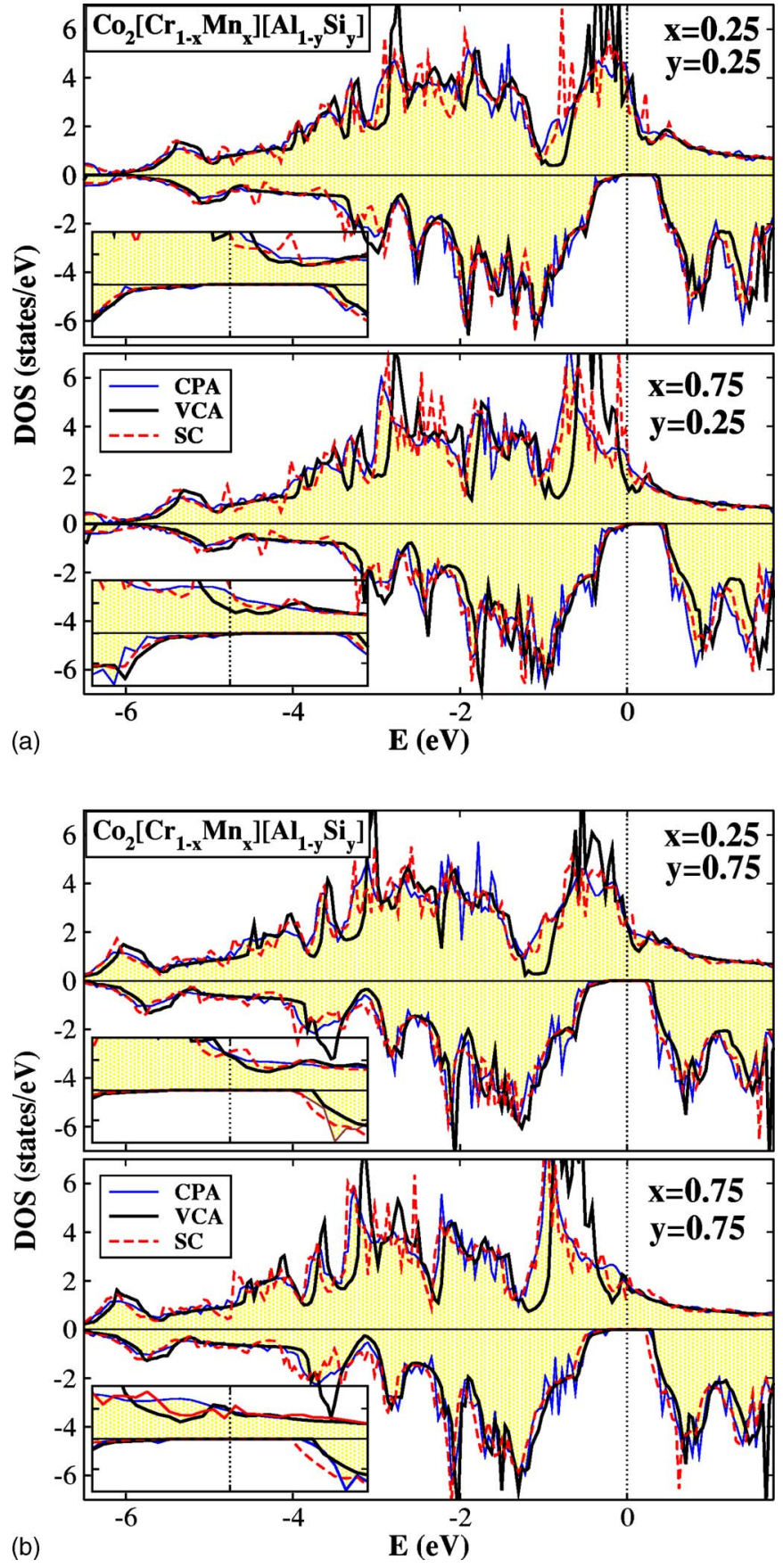

FIG. 7. (Color online) Total DOS in states/eV for four different compounds using all three CPA, VCA, and SC methods. Notice that the DOS for the SC calculations has been scaled to the elementary unit cell of four atoms, although the unit cell contains either 8 or 16 atoms.

have been scaled to one atom, and we have multiplied the DOS of the $s p$ atoms by a factor of 8 to make it visible. We do not present the VCA results since atomic DOS lack any physical meaning within this approach. Due to the very small broadening used for the DOS, CPA gives a more spiky DOS with respect to SC. The Co DOS produced by both methods is identical. In the majority spin DOS the deep in the DOS around $-1.5 \mathrm{eV}$ separates the occupied majority bonding $d$-states from the occupied antibonding majority $d$-states. As expected for the $\mathrm{Cr}$ and $\mathrm{Mn}$ atoms, the DOS presents several small differences, but overall the bands present the same shape. CPA is a mean-field theory and thus washes out any 


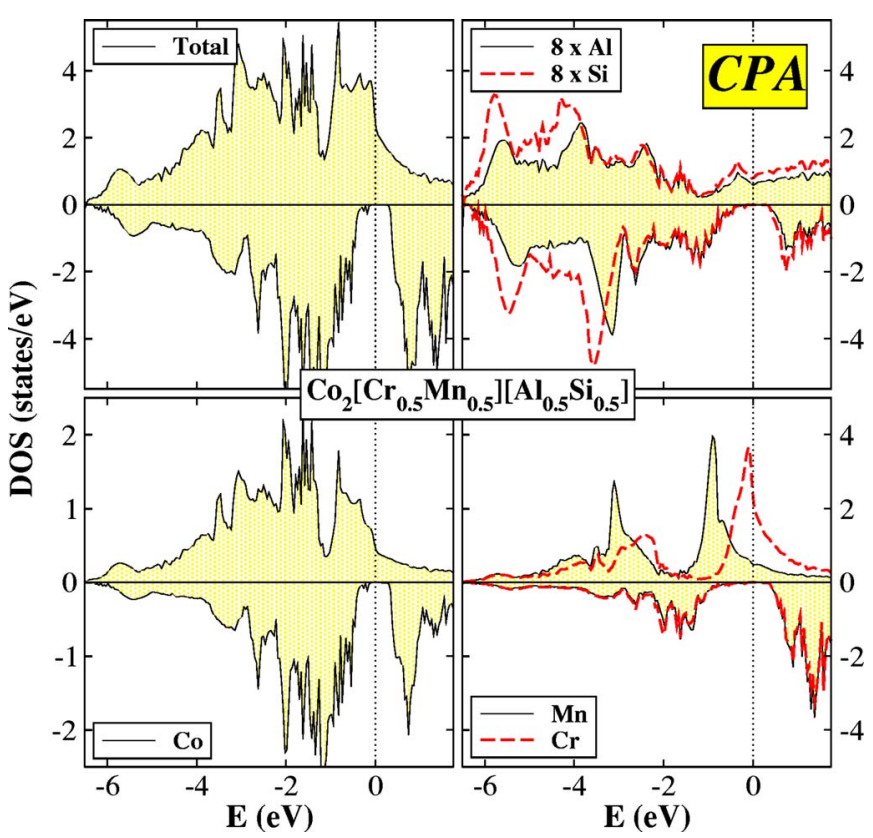

FIG. 8. (Color online) Total and atom-resolved DOS of the $\mathrm{Co}_{2}\left[\mathrm{Cr}_{0.5} \mathrm{Mn}_{0.5}\right]\left[\mathrm{Al}_{0.5} \mathrm{Si}_{0.5}\right]$ alloy using the CPA method. The $\mathrm{Al}$ and Si DOS was multiplied by a factor of 8 . All atom-resolved DOS were scaled to one atom.

small picks in the DOS. For example, we cannot distinguish between the $t_{2 g}$ and $e_{g}$ majority states of $\mathrm{Cr}$ around the Fermi level. Contrary to CPA, SC also carries short-range information and the Fermi level separates the $t_{2 g}$ and $e_{g}$ majority states of $\mathrm{Cr}$, giving a band with a double peak. Overall the bands are located at the same energy within both CPA and $\mathrm{SC}$ and have the same width, resulting in similar spin moments. The CPA and SC methods result in somewhat more important differences in the DOS of the $\mathrm{Al}$ and $\mathrm{Si}$ atoms, but this DOS is 1 order of magnitude smaller than the DOS of the transition-metal atoms, since it is mainly made up of $p$

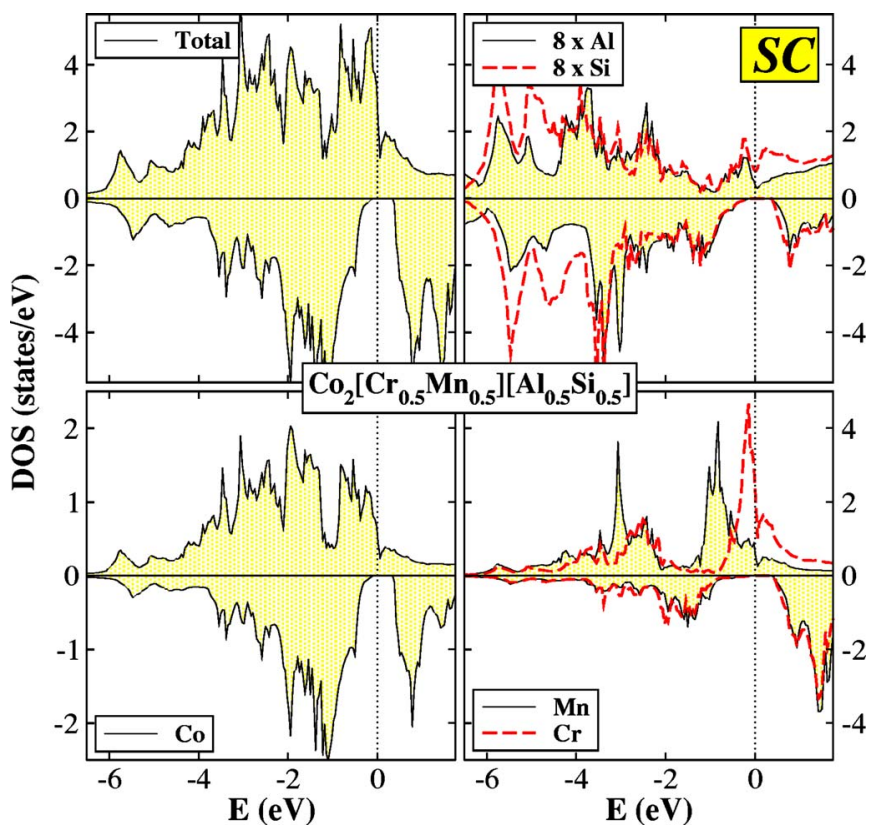

FIG. 9. (Color online) Same as Fig. 8 using the supercell construction. The total DOS was scaled as before to one elementary unit cell of four atoms. states which are spread over a wide energy range (the $s$ states are located at around $-9 \mathrm{eV}$ and are not shown here). Thus, these differences do not affect the electronic description given by the two methods, and one can safely state that short-range interactions are not important for these alloys.

\section{GAP-RELATED PROPERTIES}

In the last part of our study, we present the properties related to the gap. In Fig. 10 we present the width of the gap as a function of the concentration $y$ for constant values of $x$ in the upper left panel within all three approaches, CPA, VCA, and SC, and in the upper right panel the position of the Fermi level with respect to the left edge of the gap. In the lower panel we present the same information as a function of the concentration $x$ of the transition metal atoms, keeping the $y$ constant. Note that for the width of the gap the vertical axis goes up to $0.7 \mathrm{eV}$, while for the position of the Fermi level it rises to $0.5 \mathrm{eV}$. In Table II we present the majority density of states exactly at the Fermi level. These results show the possibility of engineering the properties related to the gap by simply changing the concentration in the low-transition metal and the $s p$ atoms. For realistic applications we need three conditions: (i) we should have a quite large gap in order to have stable half-metallicity; (ii) the Fermi level should be as close as possible to the center of the gap for the half-metallicity to be robust, since impurities and defects induce states at the edges of the gap; ${ }^{29}$ and (iii) the majority DOS at the Fermi level should be also high in order to produce significant spin-polarized current in real experiments.

The first remark concerning Fig. 10 is that all three methods give almost identical results for the width of the minority-spin gap. This enhances even further our argument in Sec. III that short-range interactions taken into account in the supercell calculations are not significant for the fullHeusler alloys. In the case of the alloys rich in $\mathrm{Si}(y=0.5$, 0.75 , and 1.0), the width of the gap is constant and around $0.6 \mathrm{eV}$, while for the compounds rich in $\mathrm{Al}(y=0.0$ and 0.25$)$ the gap is around $0.4-0.5 \mathrm{eV}$, and in the case of $\mathrm{Co}_{2} \mathrm{CrAl}$ and $\mathrm{Co}_{2} \mathrm{MnAl}$ alloys it almost vanishes. Thus, for realistic applications compounds rich in silicon are preferable, since the gap width should be large and more robust. From the lower left panel we can safely conclude that, with the exception of the compounds containing exclusively $\mathrm{Al}(y=0)$, the width of the gap is independent of the relative concentration of the $\mathrm{Cr}$ and $\mathrm{Mn}$ atoms (1-x and $x$, respectively), as the concentrations of the $\mathrm{Al}$ and $\mathrm{Si}$ atoms are kept constant. The gap is defined by the relative position of the nonbonding occupied triple-degenerated $t_{1 u}$ and unoccupied double-degenerated $e_{u}$ states, which are exclusively located at the Co sites, as shown in Ref. 20. The position of these states does not depend on the exact energy position of the $\mathrm{Cr}$ and $\mathrm{Mn}$ $d$-orbitals, and thus the width of the gap does not depend on the relative concentration of these atoms.

Now we discuss the behavior of the Fermi level. The Fermi level, contrary to the width of the gap, is determined mainly by the energy extension of the majority $p$ states of the $s p$, atoms as discussed in Ref. 20. This is clearly seen in the upper right panel of Fig. 10 where, when we keep $x$ constant, 


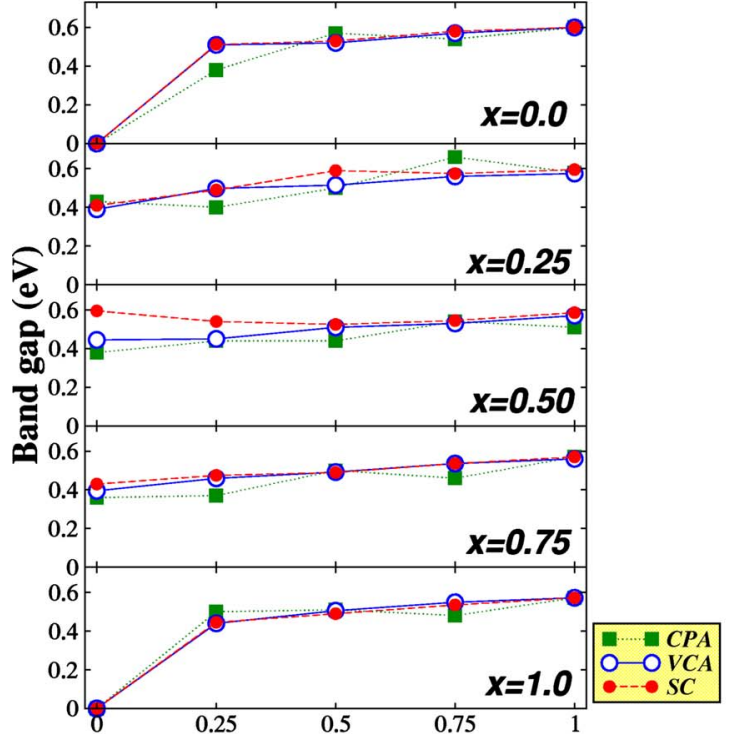

(a)

Concentration (y)

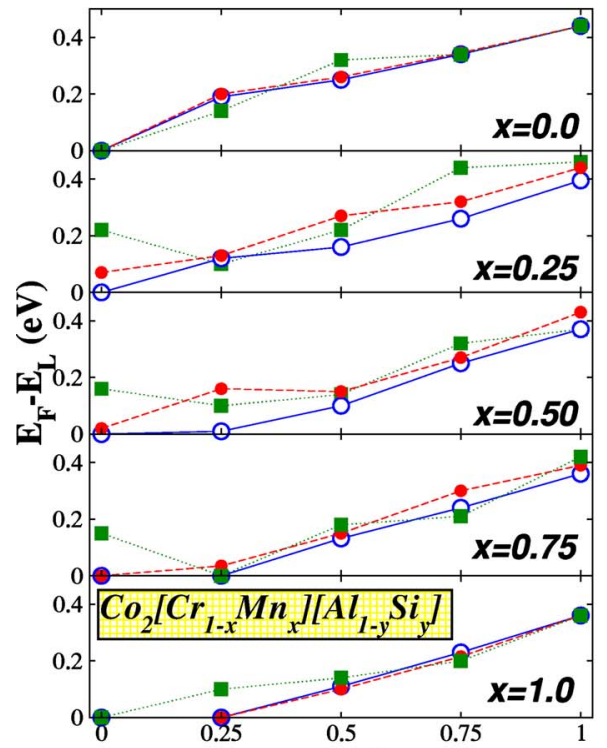

(b)

Concentration $(\mathbf{y})$

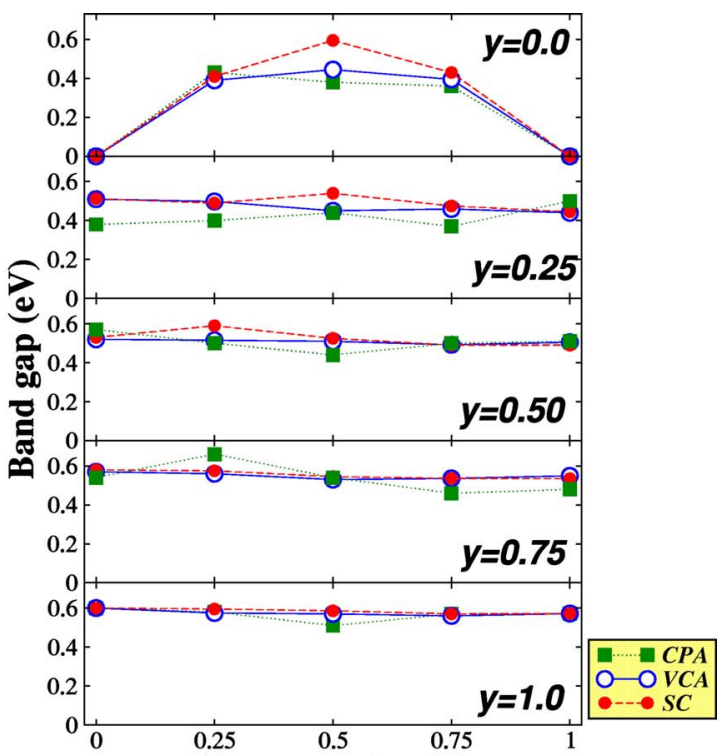

(c)

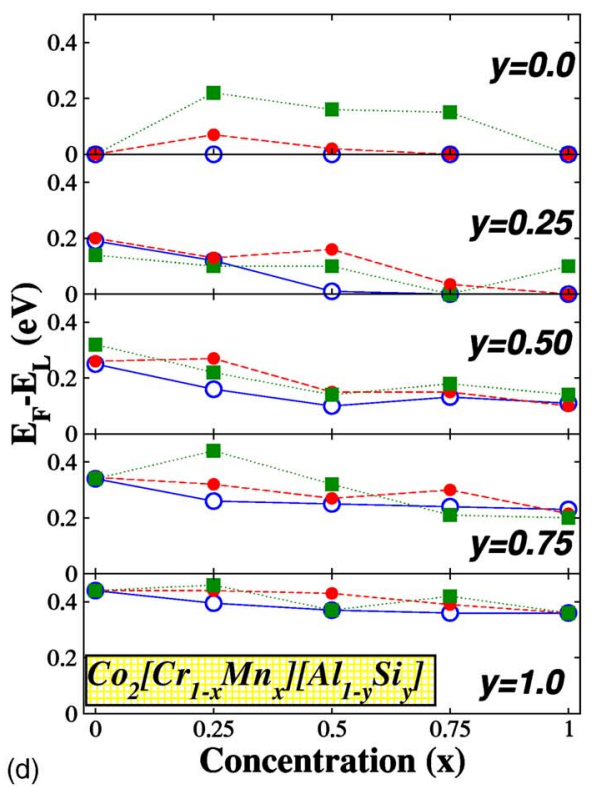

FIG. 10. (Color online) (Top) Width of the band gap (left panel) and distance of Fermi level, $E_{F}$, from the left edge of the gap, $E_{L}$, (right panel) as a function of the concentration $y$ keeping $x$ constant using all three CPA, VCA, and SC methods. (Bottom) Same as upper panel as a function of $x$ keeping $y$ constant. Note that for the band-gap width the scale of the vertical axis is different than for the position of the Fermi level.

the Fermi level is pushed higher in energy as we increase the concentration of the $\mathrm{Si}$ atoms with respect to the $\mathrm{Al}$ ones. The Fermi level is located almost at the left edge of the gap for the alloys containing only $\mathrm{Al}(y=0)$ and has a distance of $\sim 0.4 \mathrm{eV}$ from the left edge of the gap for the alloys containing only $\mathrm{Si}(y=1)$. The most interesting case are the alloys rich in $\mathrm{Si}$, with a gap of about $0.6 \mathrm{eV}$, and the ideal case should occur when the Fermi level is located at the middle of the gap with a distance of about $0.3 \mathrm{eV}$ from both edges of the gap. This is the case when $y=0.75$ and thus, at the $Z$ site $25 \%$ of the atoms are of Al chemical type and $75 \%$ of the $\mathrm{Si}$ chemical type. When $y=0.5$ and the populations of $\mathrm{Al}$ and $\mathrm{Si}$ atoms are equal the Fermi level is located at around $0.2 \mathrm{eV}$ from the left edge of the gap and thus, half-metallicity should be more affected by disorder, defects, and impurities.

Finally, we focus on the majority density of states pre- sented in Table II. All three methods give comparable results but now the SC approach gives a systematically higher value than CPA or VCA, with few exceptions. The DOS at the Fermi level is a small detail of the total DOS, and the effect of the short-range interactions is larger than when we compare the DOS over the whole energy range or the spin moments where these small details are averaged and do not manifest their presence. As we have discussed in Sec. IV, the DOS at the Fermi level is higher for the compounds rich in $\mathrm{Cr}$, since for the $\mathrm{Cr}$ atoms the Fermi level crosses the majority $d$ electrons while Mn atoms have practically all their $d$ majority states occupied. The majority DOS at the Fermi level for the compounds containing only $\operatorname{Mn}(x=1)$ is one third the value for the compounds containing only $\operatorname{Cr}(x=0)$. A second remark concerns the behavior of the majority DOS at the Fermi level with the concentration $y$ of the sp atoms 
TABLE II. Calculated spin-up (majority) density of states in states/eV at the Fermi level for the $\mathrm{Co}_{2}\left[\mathrm{Cr}_{1-x} \mathrm{Mn}_{x}\right]\left[\mathrm{Al}_{1-y} \mathrm{Si}_{y}\right]$ as a function of $x$ and $y$ concentrations with three different methods: (i) the coherent potential approximation (CPA); (ii) the virtual crystal approximation (VCA); and (iii) using supercell calculations (SC). Note that for the SC case the total DOS was scaled as before to an elementary unit cell of four atoms instead of 8 or 16 atoms of the unit cell.

\begin{tabular}{ccccc}
\hline \hline$x$ & $y$ & $\mathrm{CPA}$ & $\mathrm{VCA}$ & $\mathrm{SC}$ \\
\hline 0.00 & 0.00 & 4.861 & 4.861 & 4.861 \\
0.00 & 0.25 & 4.552 & 4.494 & 5.102 \\
0.00 & 0.50 & 3.673 & 3.196 & 3.660 \\
0.00 & 0.75 & 2.967 & 2.663 & 3.170 \\
0.00 & 1.00 & 2.318 & 2.318 & 2.318 \\
0.25 & 0.00 & 3.030 & 4.490 & 5.189 \\
0.25 & 0.25 & 3.496 & 4.201 & 3.339 \\
0.25 & 0.50 & 3.553 & 3.110 & 2.488 \\
0.25 & 0.75 & 2.271 & 2.381 & 2.544 \\
0.25 & 1.00 & 1.332 & 2.092 & 2.319 \\
0.50 & 0.00 & 4.746 & 3.861 & 4.528 \\
0.50 & 0.25 & 3.843 & 3.581 & 4.240 \\
0.50 & 0.50 & 1.906 & 2.477 & 2.804 \\
0.50 & 0.75 & 1.270 & 2.088 & 2.016 \\
0.50 & 1.00 & 1.904 & 1.861 & 1.974 \\
0.75 & 0.00 & 3.670 & 2.910 & 4.027 \\
0.75 & 0.25 & 1.543 & 2.970 & 3.051 \\
0.75 & 0.50 & 1.512 & 2.210 & 2.083 \\
0.75 & 0.75 & 1.896 & 1.851 & 1.787 \\
0.75 & 1.00 & 1.707 & 1.542 & 1.461 \\
1.00 & 0.00 & 1.510 & 1.510 & 1.510 \\
1.00 & 0.25 & 2.042 & 2.025 & 1.876 \\
1.00 & 0.50 & 1.582 & 1.556 & 1.610 \\
1.00 & 0.75 & 1.451 & 1.203 & 1.491 \\
1.00 & 1.00 & 1.222 & 1.222 & 1.222 \\
\hline \hline & & & & \\
\hline
\end{tabular}

when we keep $x$ constant. As we increase the population in $\mathrm{Si}$, the extra charge pushes the majority states lower in energy and thus, the DOS at the Fermi level deceases considerably. For the compounds containing only $\operatorname{Si}(y=1)$, the DOS at $E_{F}$ is about $40-50 \%$ of the value for the $\mathrm{Al}$ alloys $(y=0)$. As discussed previously, for realistic applications we need compounds rich in $\mathrm{Si}$ which combine a large value of the band-gap width with a Fermi level near the middle of the gap. From the present discussion it is evident that we also need our compound to be rich in $\mathrm{Cr}$ in order to ensure a high value of DOS at the Fermi level.

\section{SUMMARY AND CONCLUSIONS}

We have studied the electronic and magnetic properties of the quinternary full Heusler alloys of the type $\mathrm{Co}_{2}\left[\mathrm{Cr}_{1-x} \mathrm{Mn}_{x}\right]\left[\mathrm{Al}_{1-y} \mathrm{Si}_{y}\right]$. For our study we have employed three different approaches: the coherent potential approximation, the virtual crystal approximation, and supercell calculations to have information also for short-range interactions. All three methods gave similar results, and the local environment manifested itself only for small details of the density of states.

All three approaches predicted that the alloys under study are half-metals for all $x$ and $y$ concentrations, and their total spin moments follow the so-called Slater-Pauling be- havior: the total spin magnetic moment in the unit cell in $\mu_{B}$ equals the number of valence electrons in the unit cell minus 24. The spin moment of the transition metal atoms were found to be almost insensible to the relative concentrations of $\mathrm{Cr}$ and $\mathrm{Mn}$ elements, while the $s p$ atoms carried very small antiparallel spin moments.

All three CPA, VCA, and SC approaches yielded similar results for the properties related to the minority-spin band gap. The width of the gap is determined by states exclusively localized at the Co sites and is insensitive to the $\mathrm{Cr}$ and $\mathrm{Mn}$ concentrations and larger for the compounds rich in $\mathrm{Si}$. The Fermi level is positioned at the left edge of the gap for the alloys containing only $\mathrm{Al}$, and as we increase the concentration in $\mathrm{Si}$ it is pushed higher in energy. The majority-spin density of states at the Fermi level takes larger values for the compounds rich in $\mathrm{Cr}$ and it drops as we increase the $\mathrm{Si}$ concentration.

We have shown the possibility to engineer the properties of the half-metallic Heusler alloys by changing the concentration of the low-valent transition metal and $s p$ atoms in a continuous way. We conclude that for realistic applications the compounds rich in $\mathrm{Si}$ and $\mathrm{Cr}$ are ideal, since they combine large energy gaps (around $0.6 \mathrm{eV}$ ), robust halfmetallicity with respect to defects (the Fermi level is located near the middle of the gap), and high values of the majorityspin density of states around the Fermi level. Since such alloys are realized already experimentally in the form of thin films and multilayers and are incorporated in magnetoelectronic devices, like spin valves or magnetic tunnel junctions, we expect our results to be of interest for the community of experimentalists in the field of spintronics and to stimulate further research into these compounds.

\section{ACKNOWLEDGMENT}

The authors acknowledge the computer support of the Leibniz Institute for Solid State and Materials Research, Dresden.

${ }^{1}$ I. Žutić, J. Fabian, and S. Das Sarma, Rev. Mod. Phys. 76, 323 (2004).

${ }^{2}$ C. Felser, G. H. Fecher, and B. Balke, Angew. Chem., Int. Ed. 46, 668 (2007).

${ }^{3}$ H. Zabel, Mater. Today 9, 42 (2006).

${ }^{4}$ Half-Metallic Alloys: Fundamentals and Applications, edited by I. Galanakis and P. H. Dederichs, Lecture Notes in Physics, Vol. 676 (Springer, Berlin, 2005).

${ }^{5}$ I. Galanakis, Ph. Mavropoulos, and P. H. Dederichs, J. Phys. D 39, 765 (2006).

${ }^{6}$ I. Galanakis and Ph. Mavropoulos, J. Phys.: Condens. Matter 19, 315213 (2007).

${ }^{7}$ A. Bergmann, J. Grabis, B. P. Toperverg, V. Leiner, M. Wolff, H. Zabel, and K. Westerholt, Phys. Rev. B 72, 214403 (2005); J. Grabis, A. Bergmann, A. Nefedov, K. Westerholt, and H. Zabel, ibid. 72, 024437 (2005); J. Grabis, A. Bergmann, A. Nefedov, K. Westerholt, and H. Zabel, ibid. 72, 024438 (2005).

${ }^{8}$ J. Schmalhorst, A. Thomas, S. Kämmerer, O. Schebaum, D. Ebke, M. D. Sacher, G. Reiss, A. Hütten, A. Turchanin, A. Golzhauser, and E. Arenholz, Phys. Rev. B 75, 014403 (2007); D. Ebke, J. Schmalhorst, N.-N. Liu, A. Thomas, G. Reiss, and A. Hütten, Appl. Phys. Lett. 89, 162506 (2006); S. Kämmerer, A. Thomas, A. Hütten, and G. Reiss, ibid. 85, 79 (2004); J. Schmalhorst, S. Kämmerer, M. Sacher, G. Reiss, A. Hütten, and A. Scholl, Phys. Rev. B 70, 024426 (2004).

${ }^{9}$ N. D. Telling, P. S. Keatley, G. van der Laan, R. J. Hicken, E. Arenholz, Y. Sakuraba, M. Oogane, Y. Ando, and T. Miyazaki, Phys. Rev. B 74, 224439 (2006); Y. Sakuraba, M. Hattori, M. Oogane, Y. Ando, H. Kato, A. Sa- 
kuma, T. Miyazaki, and H. Kubota, Appl. Phys. Lett. 88, 192508 (2006); Y. Sakuraba, J. Nakata, M. Oogane, Y. Ando, H. Kato, A. Sakuma, T. Miyazaki, and H. Kubota, ibid. 88, 022503 (2006); Y. Sakuraba, T. Miyakoshi, M. Oogane, Y. Ando, A. Sakuma, T. Miyazaki, and H. Kubota, ibid. 89, 052508 (2006); Y. Sakuraba, J. Nakata, M. Oogane, H. Kubota, Y. Ando, A. Sakuma, and T. Miyazaki, Jpn. J. Appl. Phys. 44, L1100 (2005); Y. Sakuraba, J. Nakata, M. Oogane, H. Kubota, Y. Ando, A. Sakuma, and T. Miyazaki, ibid. 44, 6535 (2005).

${ }^{10}$ X. Y. Dong, C. Adelmann, J. Q. Xie, C. J. Palmström, X. Lou, J. Strand, P. A. Crowell, J.-P. Barnes, and A. K. Petford-Long, Appl. Phys. Lett. 86, 102107 (2005); H. Wang, A. Sato, K. Saito, S. Mitani, K. Takanashi, and K. Yakushiji, ibid. 90, 142510 (2007); N. Tezuka, N. Ikeda, A. Miyazaki, S. Sugimoto, M. Kikuchi, and K. Inomata, ibid. 89, 112514 (2006); K. Yakushiji, K. Saito, S. Mitani, K. Takanashi, Y. K. Takahashi, and K. Hono, ibid. 88, 222504 (2006)

${ }^{11}$ S. A. Wolf, D. D. Awschalom, R. A. Buhrman, J. M. Daughton, S. von Molnár, M. L. Roukes, A. Y. Chtchelkanova, and D. M. Treger, Science 294, 1488 (2001).

${ }^{12}$ R. A. de Groot, F. M. Mueller, P. G. van Engen, and K. H. J. Buschow, Phys. Rev. Lett. 50, 2024 (1983).

${ }^{13}$ I. Galanakis, P. H. Dederichs, and N. Papanikolaou, Phys. Rev. B 66, 134428 (2002).

${ }^{14}$ D. Jung, H.-J. Koo, and M.-H. Whangbo, J. Mol. Struct.: THEOCHEM 527, 113 (2000); B. R. K. Nanda and S. Dasgupta, J. Phys.: Condens. Matter 15, 7307 (2003); J. Kohler, S. Q. Deng, C. Lee, and M. H. Whangbo, Inorg. Chem. 46, 1957 (2007); L. Offernes, P. Ravindran, and A. Kjekshus, J. Alloys Compd. 439, 37 (2007); A. Yamasaki, L. Chioncel, A. I. Lichtenstein, and O. K. Andersen, Phys. Rev. B 74, 024419 (2006). ${ }^{15}$ M. N. Kirillova, A. A. Makhnev, E. I. Shreder, V. P. Dyakina, and N. B. Gorina, Phys. Status Solidi B 187, 231 (1995).

${ }^{16}$ K. E. H. M. Hanssen and P. E. Mijnarends, Phys. Rev. B 34, 5009 (1986); K. E. H. M. Hanssen, P. E. Mijnarends, L. P. L. M. Rabou, and K. H. J. Buschow, ibid. 42, 1533 (1990).

${ }^{17}$ P. J. Webster, J. Phys. Chem. Solids 32, 1221 (1971).

${ }^{18}$ S. Ishida, S. Fujii, S. Kashiwagi, and S. Asano, J. Phys. Soc. Jpn. 64, 2152 (1995); S. Fujii, S. Sugimura, S. Ishida, and S. Asano, J. Phys.: Condens. Matter 2, 8583 (1990).

${ }^{19}$ S. Picozzi, A. Continenza, and A. J. Freeman, Phys. Rev. B 66, 094421 (2002).

${ }^{20}$ I. Galanakis, P. H. Dederichs, and N. Papanikolaou, Phys. Rev. B 66, 174429 (2002)

${ }^{21}$ X.-Q. Chen, R. Podloucky, and P. Rogl, J. Appl. Phys. 100, 113901 (2006).

${ }^{22}$ I. Galanakis, J. Phys.: Condens. Matter 14, 6329 (2002); I. Galanakis, J. Magn. Magn. Mater. 288, 411 (2005); M. Ležaic, I. Galanakis, G. Bihlmayer, and S. Blügel, J. Phys.: Condens. Matter 17, 3121 (2005); S. J. Jenkins, Phys. Rev. B 70, 245401 (2004); S. J. Jenkins and D. A. King, Surf. Sci. 494, L793 (2001).

${ }^{23}$ K. Nagao, Y. Miura, and M. Shirai, Phys. Rev. B 73, 104447 (2006); I. Galanakis, J. Phys.: Condens. Matter 16, 8007 (2004); I. Galanakis, M. Ležaić, G. Bihlmayer, and S. Blügel, Phys. Rev. B 71, 214431 (2005); G. A. de Wijs and R. A. de Groot, ibid. 64, 020402 (2001).

${ }^{24}$ S. J. Hashemifar, P. Kratzer, and M. Scheffler, Phys. Rev. Lett. 94, 096402 (2005).

${ }^{25}$ L. Chioncel, E. Arrigoni, M. I. Katsnelson, and A. I. Lichtenstein, Phys. Rev. Lett. 96, 137203 (2006); L. Chioncel, M. I. Katsnelson, R. A. de Groot, and A. I. Lichtenstein, Phys. Rev. B 68, 144425 (2003).

${ }^{26} \mathrm{M}$. Ležaić, Ph. Mavropoulos, J. Enkovaara, G. Bihlmayer, and S. Blügel, Phys. Rev. Lett. 97, 026404 (2006).

${ }^{27}$ R. Skomski and P. A. Dowben, Europhys. Lett. 58, 544 (2002).

${ }^{28}$ S. Picozzi, A. Continenza, and A. J. Freeman, Phys. Rev. B 69, 094423 (2004).

${ }^{29}$ D. Orgassa, H. Fujiwara, T. C. Schulthess, and W. H. Butler, Phys. Rev. B 60, 13237 (1999); B. Alling, S. Shallcross, and I. A. Abrikosov, ibid. 73 , 064418 (2006); J. J. Attema, C. M. Fang, L. Chioncel, G. A. de Wijs, A. I. Lichtenstein, and R. A. de Groot, J. Phys.: Condens. Matter 16, S5517 (2004); M. J. Carey, T. Block, and B. A. Gurney, Appl. Phys. Lett. 85, 4442 (2004).

${ }^{30}$ K. Özdoğan, E. Şaşıoğlu, and I. Galanakis, Phys. Status Solidi (RRL) 1, 184 (2007).
${ }^{31}$ I. Galanakis, Phys. Rev. B 71, 012413 (2005); M. Sargolzaei, M. Richter, K. Koepernik, I. Opahle, H. Eschrig, and I. Chaplygin, ibid. 74, 224410 (2006).

${ }^{32}$ I. Galanakis, K. Özdoğan, B. Aktaş, and E. Şaşıŏlu, Appl. Phys. Lett. 89, 042502 (2006); K. Özdoğan, E. Şașığlu, B. Aktaș, and I. Galanakis, Phys. Rev. B 74, 172412 (2006).

${ }^{33}$ T. Block, M. J. Carey, D. A. Gurney, and O. Jepsen, Phys. Rev. B 70, 205114 (2004)

${ }^{34}$ K. Özdoğan, I. Galanakis, E. Şaşıŏglu, and B. Aktaş, Phys. Status Solidi (RRL) 1, 95 (2007).

${ }^{35}$ K. Özdoğan, K. I. Galanakis, E. Şaşıoğlu, and B. Aktaş, Solid State Commun. 142, 492 (2007).

${ }^{36}$ K. Özdoğan, I. Galanakis, E. Şaşıŏlu, and B. Aktaş, J. Phys.: Condens. Matter 18, 2905 (2006); E. Sașioğlu, L. M. Sandratskii, and P. Bruno, ibid. 17, 995 (2005); S. Ishida, S. Asano, and J. Ishida, J. Phys. Soc. Jpn. 53, 2718 (1984); R. Weht and W. E. Pickett, Phys. Rev. B 60, 13006 (1999).

${ }^{37}$ I. Galanakis, K. Özdoğan, E. Şaşığlu, and B. Aktaș, Phys. Rev. B 75, 172405 (2007); I. Galanakis, K. Özdoğan, E. Şaşıoğlu, and B. Aktaş, ibid. 75, 092407 (2007); H. van Leuken and R. A. de Groot, Phys. Rev. Lett. 74, 1171 (1995); S. Wurmehl, H. C. Kandpal, G. H. Fecher, and C. Felser, J. Phys.: Condens. Matter 18, 6171 (2006).

${ }^{38}$ E. Şaşığlu, L. M. Sandratskii, P. Bruno, and I. Galanakis, Phys. Rev. B 72, 184415 (2005).

${ }^{39}$ Y. Kurtulus, R. Dronskowski, G. D. Samolyuk, and V. P. Antropov, Phys. Rev. B 71, 014425 (2005).

${ }^{40}$ I. Galanakis, J. Phys.: Condens. Matter 16, 3089 (2004).

${ }^{41}$ K. Özdoğan, B. Aktaş, I. Galanakis, and E. Şaşığlu, J. Appl. Phys. 101, 073910 (2007).

${ }^{42}$ V. N. Antonov, H. A. Dürr, Yu. Kucherenko, L. V. Bekenov, and A. N. Yaresko, Phys. Rev. B 72, 054441 (2005).

${ }^{43}$ Y. Miura, K. Nagao, and M. Shirai, Phys. Rev. B 69, 144413 (2004).

${ }^{44}$ B. Balke, G. H. Fecher, H. C. Kandpal, C. Felser, K. Kobayashi, E. Ikenaga, J.-J. Kim, and S. Ueda, Phys. Rev. B 74, 104405 (2006).

${ }^{45}$ H. M. Kandpal, G. H. Fecher, C. Felser, and G. Schönhense, Phys. Rev. B 73, 094422 (2006)

${ }^{46}$ S. Wurmehl, G. H. Fecher, H. C. Kandpal, V. Ksenofontov, C. Felser, and H.-J. Lin, Appl. Phys. Lett. 88, 032503 (2006).

${ }^{47}$ K. Koepernik, B. Velicky, R. Hayn, and H. Eschrig, Phys. Rev. B 58, 6944 (1998).

${ }^{48}$ K. Koepernik and H. Eschrig, Phys. Rev. B 59, 1743 (1999).

${ }^{49}$ S. H. Vosko, L. Wilk, and N. Nusair, Can. J. Phys. 58, 1200 (1980); P. Hohenberg and W. Kohn, Phys. Rev. 136, B864 (1964); W. Kohn and L. J. Sham, ibid. 140, A1133 (1965).

${ }^{50} \mathrm{P}$. J. Webster and K. R. A. Ziebeck, in Alloys and Compounds of d-Elements with Main Group Elements, Part 2, edited by H. R. J. Wijn, Landolt-Boörnstein, New Series, Group III, Vol. 19/c (Springer-Verlag, Berlin, 1988), pp. 75-184.

${ }^{51}$ K. R. A. Ziebeck and K.-U. Neumann, in Magnetic Properties of Metals, edited by H. R. J. Wijn, Landolt-Börnstein, New Series, Group III, Vol. 32/c (Springer, Berlin, 2001), pp. 64-414.

${ }^{52}$ J. A. Blackman, D. M. Esterling, and N. F. Berk, Phys. Rev. B 4, 2412 (1971).

${ }^{53}$ A. D. Rata, H. Braak, D. E. Bürgler, S. Cramm, and C. M. Schneider, Eur. Phys. J. B 52, 445 (2006); M. Kallmayer, H. Schneider, G. Jakob, H. J. Elmers, K. Kroth, H. C. Kandpal, U. Stumm, and S. Cramm, Appl. Phys. Lett. 88, 072506 (2006); S. V. Karthik, A. Rajanikanth, Y. K. Takahashi, T. Okhubo, and K. Hono, ibid. 89, 052505 (2006); G. H. Fecher, H. C. Kandpal, S. Würmehl, J. Morais, H.-J. Lin, H.-J. Elmers, G. Schönhense, and C. Felser, J. Phys.: Condens. Matter 17, 7237 (2005); R. Y. Umetsu, K. Kobayashi, A. Fujita, K. Oikawa, R. Kainuma, K. Ishida, N. Endo, K. Fukamichi, and A. Sakuma, Phys. Rev. B 72, 214412 (2005); K. Kobayashi, R. Y. Umetsu, R. Kainuma, K. Ishida, T. Oyamada, A. Fujita, and K. Fukamichi, Appl. Phys. Lett. 85, 4684 (2004); H. J. Elmers, G. H. Fecher, D. Valdaitsev, S. A. Nepijko, A. Gloskovskii, G. Jakob, G. Schönhense, S. Wurmehl, T. Block, C. Felser, P.-C. Hsu, W.-L. Tsai, and S. Cramm, Phys. Rev. B 67, 104412 (2003)

${ }^{54}$ T. Marukame, T. Ishikawa, K.-I. Matsuda, T. Uemura, and M. Yamamoto, Appl. Phys. Lett. 88, 262503 (2006); T. Marukame, T. Kasahara, K. Matsuda, T. Uemura, and M. Yamamoto, Jpn. J. Appl. Phys. 44, L521 (2005). ${ }^{55}$ R. Kelekar and B. M. Klemens, Appl. Phys. Lett. 86, 232501 (2005). 